\title{
Management of Aplastic Anaemia in a 15-year Old Boy with Down Syndrome in a Low Resource Setting
}

\author{
Tallal Hussain ${ }^{1 *}$, Mutwali AM Hussain ${ }^{2}$, Atif A Saad ${ }^{3}$ and Galal Babekir ${ }^{3}$ \\ ${ }^{1}$ Specialist Registrar in Pediatrics, Pediatric Intensive Care Unit, Leeds General Infirmary, UK \\ ${ }^{2}$ Professor of Pediatrics and Child Health and Consultant Haematologist, University of Science and Technology, Sudan \\ ${ }^{3}$ Consultant Pediatrician, Albuluk Specialised Hospital for Children, Sudan
}

*Corresponding author: Tallal Hussain, Specialist Registrar in Pediatrics,

Received Date: August 29, 2019

Pediatric Intensive Care Unit, Leeds General Infirmary, UK.

Published Date: September 06, 2019

\section{Summary}

We present a case report of idiopathic aplastic anaemia (AA) in a 15-year old Sudanese boy with Down syndrome (47XY, 21+ in all metaphases). AA is rare in Down syndrome with 10 cases published in the literature. The patient presented with a history of mouth mucosal bleeding, pallor and fever. His full blood count (FBC) showed pancytopenia and a bone marrow biopsy confirmed the finding of a hypocellular bone marrow. A diagnosis of AA in this child with Down syndrome was made based on the presentation and investigations. Other differentials were considered and excluded. He was treated with regular packed red blood cell and platelet transfusions because of the lack of facilities for bone marrow transplant (BMT). He was started on Deferasirox (Exjade) for high serum ferritin. He passed away four weeks after starting Exjade with severe sepsis. The rationale for reporting this case is to highlight AA in the differential diagnosis of anemia in a child with Down's syndrome and to describe the restricted options available in a resource-poor setting. It also highlights the possible role of Deferasirox (Exjade) for treatment of pancytopenia in AA.

\section{Background}

AA is rare in children with Down syndrome and the exact number of case reports is not clear. However, between 1967-2017 there were only a few cases reported in the world literature. Because of its rarity, there are a few publications about its management which is a challenge in a low-income setting.

\section{Presentation}

A 15-year old male with Down syndrome $(47 \times \mathrm{Y}, 21+$ in all metaphases) was admitted to his local hospital with a history of mucosal mouth bleeding, pallor and fever. His past medical history was unremarkable apart from minor upper respiratory tract infections. There was no family history of bleeding disorders or hereditary anaemias. Furthermore, there was no history of exposure to any drug or infectious agent that may result in bone marrow failure. Physical examination showed the usual features of Down syndrome. His weight and height were both on the 50th centile (for age, sex and condition). There was marked pallor with bruising and purpuric rash in both upper and lower limbs. There was no palpable organomegaly or lymphadenopathy. His vital signs and cardiovascular examination were normal.

\section{Investigations}

Blood tests demonstrated marked pancytopenia with hemoglobin of $59 \mathrm{~g} / \mathrm{L}$ (reticulocytes $0.3 \%$ ), white cell count (WCC) $2 \times 109 / \mathrm{L}$ (neutrophils $0.4 \times 109 / \mathrm{L}$, lymphocytes $1.5 \times 109 / \mathrm{L}$ ) and platelets $6 \times 109 / \mathrm{L}$. His bone marrow aspiration and trephine biopsy confirmed hypocellular bone marrow with absent megakaryocytes, depressed erythropoiesis and granulopoiesis. His blood culture revealed no growth on admission. His abdominal ultrasound scan (USS) did not show lymphadenopathy or anything suggestive of a solid tumour. Screening for HIV, Epstein-Barr virus (EBV), Hepatitis $\mathrm{B}$ and $\mathrm{C}$ were all negative. His renal and liver function tests were both normal. His TSH was within normal limit.

\section{Differential Diagnosis}

A diagnosis of AA in this child with Down syndrome was made based on the presentation and investigations. No contributing factors for AA were identified. The diagnosis of haematological malignancy was considered but was excluded by the absence of lymphadenopathy or organomegaly on examination and by the findings on the blood film, bone marrow aspiration and trephine biopsy. The possibility of an occult solid tumour was entertained 
but there was no evidence of this on abdominal USS, bone marrow aspiration or biopsy. We did not feel the need for any other tests.

\section{Treatment}

He was started on broad spectrum antibiotics for suspected sepsis on admission. The patient was initially managed conservatively because none of the standard Immunosuppressive Therapy (IST) relevant to his condition were available. He was transfused 17 times with packed red cell transfusions (pRBC) and he required 294 pools of platelets over a period of 12 months. He was started on Exjade because his serum ferritin reached a peak of $2036 \mathrm{mcg} / \mathrm{L}$.

\section{Outcome and Follow-Up}

The patient passed away seven months after starting Exjade with severe sepsis.

\section{Discussion}

Haematological problems in children with Down syndrome include myeloproliferative disorder of childhood, leukaemia, myelodysplastic syndrome and AA [1]. The first case of idiopathic AA in Down syndrome was published by Erdogan et al in 1967 reporting a 17-year old boy who presented with severe epistaxis [2]. In 1981 Weinblatt, et al. [3] claimed their report of the first case of AA in Down syndrome [3]. McWilliams et al reported another case of AA in 1982 in a 27-month old black girl with Down syndrome who presented 9 months after repair of an endocardial cushion defect [4]. In 2003, Pavithran, et al. [1] reported AA in a 2-year old girl with Down syndrome who responded to oxymetholone. She required less pRBC transfusions but there was no improvement in the white cell or platelet counts [1]. In 2011, Gathwala et al. [5] published a case of AA in a child with Down syndrome and hypothyroidism. The pancytopenia resolved after treatment with thyroxine [5].

Our report describes a boy with idiopathic AA and its management in a child with Down syndrome in a low resource setting. The definitive treatment for AA is BMT [6]. In patients who lack an HLA-matched sibling donor, IST with antithymocyte globulin and cyclosporine A appears to be a safe and reasonable treatment [7]. There is now evidence that Exjade may have a role in the management of AA. In the EPIC study, 24 out of 72 patients with
AA and iron overload were treated with Exjade without concomitant IST. Eleven of these patients became transfusion independent $[8,9]$. The mechanisms for this response remain unclear and further research is required.

\section{Learning Points / Take Home Messages}

1. Though rare, AA should be considered in any child with Down syndrome who presents clinically with pallor.

2. Some cases of AA in Down syndrome can be cured by treating the possible cause e.g. hypothyroidism.

3. Exjade and IST should be considered in the management of Down syndrome and AA in the absence of facilities for BMT.

4. It is not clear whether the chromosomal disorder associated with Trisomy 21 is a predisposing factor for AA. Further reviews and research are required.

\section{Acknowledgement}

None.

\section{Conflict of Interest}

None.

\section{References}

1. Keechilat Pavithran, Navamony Laila Raji (2003) Aplastic anemia in Down's syndrome. American Journal of Hematology 73(3): 213-213.

2. Erdoğan G, Aksoy M, Dinçol K (1967) A case of idiopathic aplastic anaemia associated with trisomy-21 and partial endoreduplication. Acta Haematol 37(2): 137-142.

3. Weinblatt ME, Higgins G, Ortega JA (1981) Aplastic anemia in Down's syndrome. Pediatrics 67(6): 896-897.

4. McWilliams, Nancy L Dunn (1982) Aplastic anemia and Down's syndrome. Pediatrics 69(4): 501-502.

5. Gathwala G, Dalal P, Dalal JS, Choudhry O (2011) Transient aplastic anemia in Down's syndrome - a rare association. Eur J Med Genet 54(3): 341-342.

6. Dezern AE, Brodsky RA (2011) Clinical management of aplastic anemia. Expert Rev Hematol 4(2): 221-230.

7. Suzuki K, Muramatsu H, Okuno Y, Narita A, Hama A, et al. (2016) Immunosuppressive therapy for patients with Down syndrome and idiopathic aplastic anemia. Int J Hematol 104(1): 130-133.

8. Lee JW (2013) Hematologic responses in patients with aplastic anemia treated with deferasirox: a post hoc analysis from the EPIC study. Haematologica 98(7): 1045-1048. 